Historic, Archive Document

Do not assume content reflects current scientific knowledge, policies, or practices. 


\section{LAWN SEED}

For Fall Or Spring Shipment

Our lawn mixtures are the result of many years experience in blending to ensure the best permanent green lawn, and are composed of the finest Domestic and Imported turf grasses carefully recleaned to reduce the weed and chatf content to a minimum.

A high quality lawn grass mixture for every requirement.

GLOBE BRAND - Extra heavy superfine.

In cartons and $5 \mathrm{lb}$. bags

In bulk

DICKINSON CLUB GREEN - Our special blend of selected imported and native grasses.

In cartons and 5 lib. bags

ln bulk

ACE BRAND - A blend of quick growing native grasses (Contains White Clover)

In bulk

EVERGREEN - Good standard quality, satisfactory for general purposes. (Centains White Clover)

In cartons and $5 \mathrm{lb}$. bags.

In bulk

SHADY SPOT - Adapted to shaded places and moist soils.

In cartons and $5 \mathrm{lb}$. bags......

In bulk

all per 100 lbs., f. o. b. Chicago.

We offer a complete line of unblended fancy grasses for lawns and golf courses. Note prices on other side.

\section{Use Post This Card}

It is printed here for your convenience

It Gets Prompt Attention

Cut out along dotted line

\section{Business Reply Card}

No Postage Stamp Neceseary is Mailed la The United Suates

2c.-POSTAGE WILL BE PAID BY-

\section{THE ALBERT DICKINSON CO.}

\section{Lock Drawer 788}




\section{BAYER-SEMESAN SEED DISINFECTANTS \\ CERESAN}

A Dust Disiafectnat for Whert, Nures Dariey, whisg Cereale and Cotton.

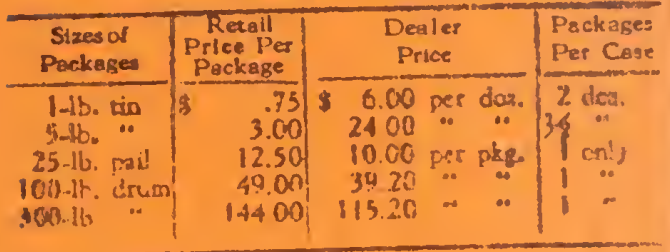

\section{SEMESAN}

A Ginesal Diwninceant for Vegetab!e and Plower beesk or Bulbs, and Certain Plant Drineases.

\begin{tabular}{|c|c|c|c|}
\hline $\begin{array}{l}\text { Slzes of } \\
\text { Packazes }\end{array}$ & $\begin{array}{l}\text { Retall } \\
\text { Price Pet } \\
\text { Parkage }\end{array}$ & $\begin{array}{l}\text { Dealer } \\
\text { Price }\end{array}$ & $\begin{array}{l}\text { Packakes } \\
\text { Per Case }\end{array}$ \\
\hline 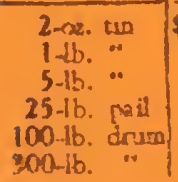 & $\begin{array}{r}.50 \\
2.75 \\
13.00 \\
51.25 \\
200.00 \\
585.00\end{array}$ & 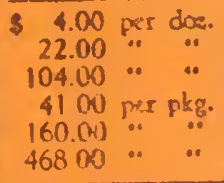 & $\left\{\begin{array}{cc}3 & \text { dos. } \\
2 & \cdots \\
1 / 3 & \cdots \\
1 & \text { ouly } \\
1 & \infty \\
1 & \cdots\end{array}\right.$ \\
\hline
\end{tabular}

SEMESAN JR.

A Dust Dusunfectant for Seed Corn

\begin{tabular}{|c|c|c|c|c|}
\hline $\begin{array}{l}\text { Sizes of } \\
\text { Packeges }\end{array}$ & $\begin{array}{l}\text { Retall } \\
\text { Price Per } \\
\text { Packsge }\end{array}$ & $\begin{array}{l}\text { Dealer } \\
\text { Price }\end{array}$ & & $\begin{array}{l}\text { Pachanes } \\
\text { Per Case }\end{array}$ \\
\hline 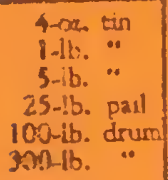 & $\begin{array}{r}.50 \\
1.75 \\
8.00 \\
31.25 \\
120.00 \\
345.00\end{array}$ & $\begin{array}{l}4.00 \text { per } \\
14.00 \cdots \\
64.00 \cdots \\
25.00 \text { per } \\
96.00 \cdots \\
276.00 \cdots\end{array}$ & $\begin{array}{c}\text { doa. } \\
\text {.. } \\
\text { pkg. } \\
.0\end{array}$ & $\begin{array}{ll}9 & d x \\
2 & = \\
10 & 00 \\
1 & \infty\end{array}$ \\
\hline
\end{tabular}

\section{NU - GREEN}

A Grass Disinfectaut for Brown-Patch Prevention Contral.

\begin{tabular}{|c|c|c|c|}
\hline $\begin{array}{l}\text { Slzes of } \\
\text { Packanes }\end{array}$ & $\begin{array}{l}\text { Retall } \\
\text { Price Per } \\
\text { Prekage } \\
\end{array}$ & $\begin{array}{l}\text { Dealer } \\
\text { Price }\end{array}$ & $\begin{array}{l}\text { Pecksges } \\
\text { Per Cass }\end{array}$ \\
\hline 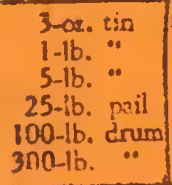 & $\begin{array}{r}.50 \\
2.00 \\
9.00 \\
33.75 \\
130.00 \\
375.00\end{array}$ & 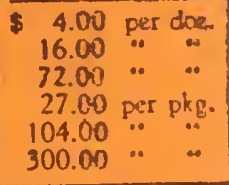 & $\left\{\begin{array}{cc}3 & d a \\
2 & * \\
1 / 2 & * \\
1 & \text { anl } \\
1 & \cdots \\
1 & \infty\end{array}\right.$ \\
\hline
\end{tabular}

\section{SEMESAN BEL}

AB Instantaneous Dip Disinfectant for Seed Potavece

\begin{tabular}{|c|c|c|c|}
\hline $\begin{array}{c}\text { Sizes of } \\
\text { Packages }\end{array}$ & $\begin{array}{c}\text { Retail } \\
\text { Price Per } \\
\text { Packsge } \\
\end{array}$ & $\begin{array}{c}\text { Dealor } \\
\text { Prtee }\end{array}$ & $\begin{array}{l}\text { Pockage } \\
\text { Per Case }\end{array}$ \\
\hline $\begin{array}{l}\text { 4-oe tin } \\
\text { 1.ib. } \\
5 \text {-ib. } . \\
\text { 25-lb. pail } \\
100-1 b . \text { drum } \\
\text { 100-lb. }\end{array}$ & $\begin{array}{r}.50 \\
1.75 \\
8.00 \\
31.25 \\
120.00 \\
345.00\end{array}$ & 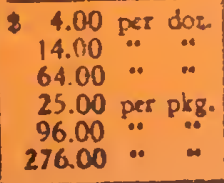 & $\mid \begin{array}{cc}3 & \text { dac } \\
2 & \infty \\
1 / 2 & \infty \\
1 & \text { only } \\
1 & \infty \\
1 & \infty\end{array}$ \\
\hline
\end{tabular}


General Offices, Warehouses and Refineries - 35th St. at Californis Ave. Postal Address-Lock Drawer 788. Long Distance Telephone-Lafayette 3000 CHICAGO, ILL., U. S. A.

BRANCH OFFICES

MINNEAPOLIS NEW YORK 